\title{
A Metadata and Z Score-based Load-Shedding Technique in IoT-based Data Collection Systems
}

\author{
Mario José Diván \\ Data Science Research Group, \\ Economy School, National University of La Pampa, \\ Coronel Gil 353, $1^{\text {st }}$ floor, Santa Rosa, CP6300, Argentina. \\ Corresponding author: mjdivan@eco.unlpam.edu.ar

\section{María Laura Sánchez-Reynoso} \\ Data Science Research Group, \\ Economy School, National University of La Pampa, \\ Coronel Gil 353, $1^{\text {st }}$ floor, Santa Rosa, CP6300, Argentina. \\ E-mail: mlsanchezreynoso@eco.unlpam.edu.ar
}

(Received July 14, 2020; Accepted August 22, 2020)

\begin{abstract}
The Internet-of-Things (IoT) has emerged as an alternative to communicate different pieces of technology to foster the distributed data collection. The measurement projects and the Real-time data processing are articulated to take advantage of this environment, fostering a sustainable data-driven decision making. The Data Stream Processing Strategy (DSPS) is a Stream Processing Engine focused on measurement projects, where each concept is previously agreed through a measurement framework. The Measurement Adapter (MA) is a component whose responsibility is to pair each metric's definition from the measurement project with data sensors to transmit data (i.e., measures) with metadata (i.e., tags indicating the data meaning) together. The Gathering Function (GF) receives and derivates data for its processing from each MA, while it implements load-shedding (LS) techniques based on Metadata to avoid a processing collapse when all MAs informs jointly and frequently. Here, a Metadata and Z-score based load-shedding technique implemented locally in the MA is proposed. Thus, the load-shedding is located at the same data source to avoid data transmission and saving resources. Also, an incremental estimation of average, deviations, covariance, and correlations are implemented and employed to calculate the Z-scores and to data retain/discard selectively. Four simulations discrete were designed and performed to analyze the proposal. Results indicate that the local LS required only $24 \%$ of the original data transmissions, a minimum of $18.61 \mathrm{~ms}$ as the data lifespan, while it consumes $890.26 \mathrm{~KB}$. As future work, other kinds of dependencies analysis will be analyzed to provide local alternatives to LS.
\end{abstract}

Keywords- Z-score, Incremental load-shedding, Reliability, Real-time, Data systems, IoT.

\section{Introduction}

The Internet-of-Things (IoT) has allowed the emerging of a set of alternatives oriented to implement data collection based on heterogeneous devices. In other words, such alternatives are composed of different kinds of tiny and cheap devices able to collect data to inform them as soon as possible to data processors through different communication technologies (Nour et al., 2019).

The fact of fostering the connectivity among every technological piece has increased the heterogeneity. On the one hand, it could be viewed as an advantage due to the possibility to generate a collecting strategy as wide as possible. On the other hand, the heterogeneity increases the complexity of a system derived from factors such as the required data interoperability, considering the syntactical and semantical perspectives for the data processing (Chen et al., 2019). 
International Journal of Mathematical, Engineering and Management Sciences

Vol. 6, No. 1, 363-382, 2021

https://doi.org/10.33889/IJMEMS.2021.6.1.023

In this context, the data streaming paradigm emerged as a natural alternative to process the data as soon as they arrive, focusing on the data processing and analysis around situations that required a real-time answer, for example, in stock markets, online fraud detection, among others. However, the heterogeneity viewed as an advantage from a certain perspective, it would imply the necessity of homogenizing the data transmission jointly with the data accuracy, precision, and their meanings to integrate each technological piece into the data collection system (Cardellini et al., 2019; Izadpanah et al., 2019).

Some characteristics of the heterogeneous data sources producing data streams are particularly important to be highlighted around the data processing: a) One-time reading: each datum needs to be read ideally one time due to always a new datum would be arriving and replacing to the previous one. In other words, the use of additional time for a new reading of a past datum does not have sense because always the updated version of it is close to arriving, b) Limited Resources: the data are analyzed by the processor just using the available resources at the moment in which they arrive, c) Raw data: the datum is processed such as it is, it comes directly from the data source, d) Arriving rate: the datum arrives at its processing depending on the data generation rate related to the data source, the data processor has not any influence on the data source (Fernández-Rodríguez et al., 2017; Traub et al., 2017).

When the data arriving rate from data sources does not exceed the processor's processing rate all it will go on wheels. Though the data processor has no control over data sources and the arriving rates could change randomly exceeding the processing rate. When such a situation is configured, the load-shedding techniques emerge as an alternative to produce a selective discarding, retaining data based on some criteria instead of simply discard them. In this sense, the final aim is oriented to keep the system availability, which is essential in terms of its reliability (Zhao et al., 2020).

The Data Stream Processing Strategy (DSPS) is a workflow implemented on a Stream Processing Engine (SPE) to automatize measurement projects based on distributed measurement devices. The underlying concepts associated with the measurement are based on a Measurement and Evaluation (MandE) framework, which provides a meaning homogeneity to interchange data between heterogeneous devices. Each measurement project is structured and specified using the MandE framework to describe the objective, concept under monitoring, concept's properties, metrics to be implemented, associated instruments, among other aspects. The definition is completely contained in a single XML (eXtensible Markup Language) file that is shared among all the components of DSPS. In this way, each component knows the unit, scale, method, expected values range, among other aspects used for obtaining a measure from the concept under monitoring through a given instrument (Diván and Sánchez Reynoso, 2020b).

The data communication in DSPS is established between the Measurement Adapter (MA) and Gathering Function (GF). On the one hand, MA is located on a mobile device and it is responsible to deal with sensors, collect their data, associate data with its meaning based on the project definition, and inform them to GF. In this way, MA is an intermediary between GF and the sensors allowing homogenizing each measure following the measurement project specification. On the other hand, GF receives data coming from each MA for processing and analyzing them in realtime. When there is a risk of exceeding the GF's processing rate, DSPS automatically activates in GF a Metadata-based load-shedding technique which allows retaining only those data related to the most important properties under monitoring, employing for such an aim the project specification (Diván and Sánchez Reynoso, 2020a). However, this requires that data are transmitted from MA to 
International Journal of Mathematical, Engineering and Management Sciences

Vol. 6, No. 1, 363-382, 2021

https://doi.org/10.33889/IJMEMS.2021.6.1.023

GF, and eventually GF will analyze whether data are processed or not. In case of the data are discarded (totally or partially depending on the associated metric), a certain number of resources were consumed for transmitting unnecessary or not so important data.

Fog computing proposes to carry out the data processing and provide answers using local data and so close to users as possible to decrease the latency and optimize resources. In other words, each time that a certain data volume needs to be informed, a determined quantity of resources is consumed to reach such an aim, while they could be used to provide a local answer. Thus, the optimization of data transmission jointly with the possibility to incorporate a local functionality useful for end users represents a promising perspective of this kind of paradigm (Ghobaei-Arani et al., 2020).

As contributions, a) A local load-shedding functionality is incorporated in MA: New load-shedding functionality is incorporated into the MA component, allowing regulating the data volume based on the measurement project definition before its transmission. The local data buffer will activate or not the temporal data transmission barrier used to provide a proof-of-life, depending on the loadshedding technique is activated or not. Thus, when the load-shedding technique is enabled, the only data transmission policy allowed from MA, it is associated with the online data change detection, b) Online covariance estimators are incorporated into the local data buffer: Each time that a datum is added in the data buffer, incremental estimations about the arithmetic mean and deviations for each metric jointly with the covariance matrix are computed. This is especially useful to get Zscores in case of being necessary (e.g., considerable differences of magnitudes between variables), or to detect potential dependencies between variables at the source, c) A Z Score and Metadatabased load-shedding technique is proposed: Each metric has a given weight defined in the project definition as an indication of its relative importance. Through the joint use of the metric's weightings and their $\mathrm{Z}$ scores, only a subset of data vectors will be retained. The retained vectors are locally kept in the data buffer to be analyzed and eventually transmitted when a data change is detected, d) The pabmmCommons library is updated and released on GitHub: All the techniques here described are implemented into the pabmmCommons library, which is freely accessible on GitHub under the terms of the Apache 2.0 license, e) Applicability patterns are provided: Two discrete simulations are implemented on the data buffer to provide applicability patterns around the obtained time and memory consumption.

The rest of this article is organized in 6 sections as follows. Section 2 describes some related works. Section 3 describes the articulation between the data buffer and the estimators. Section 4 describes the load-shedding technique and its data retention policy. Section 5 describes the simulation methodology through a Business Process Model and Notation (BPMN) diagram. Also, it analyzes the obtained results from simulation to describe applicability patterns but also their associated limitations. Section 6 outlines some conclusions and future works.

\section{Related Works}

In Cardellini et al. (2019), authors propose an interesting load-shedding methodology to retain or discard events according to a given set of policies. As a similarity, authors use metadata to analyze each event before deciding about retaining it or not, while our strategy uses the measure metadata from project definition to analyze each data in context before discarding it or not. As a difference, this proposal acts on the data processor, while our proposal analyzes the data directly at the data source, into the same measurement adapter with direct contact with sensors. In this way, the data is retained or not before it consumes resources for any intent of transmission. 
International Journal of Mathematical, Engineering and Management Sciences

Vol. 6, No. 1, 363-382, 2021

https://doi.org/10.33889/IJMEMS.2021.6.1.023

In Slo et al. (2019), authors describe the building of a probabilistic model that can analyze the events present in the windows jointly with its mutual influence to determine what event to retain. In this sense, the authors focus on the interaction between events instead of the analysis of each isolated event. As a similarity, our proposal updates incrementally the covariance matrix to detect dependence between metrics before retaining or discarding. As a difference, the variation estimation and measure analysis are performed as close to data sources as possible, before the data is transmitted.

Table 1 incorporates a synthesis of related works here introduced, focusing on their aims and proposed approaches.

Table 1. A comparative perspective of the aim and approach of each related work.

\begin{tabular}{|c|c|c|}
\hline Article & Aim & Approach \\
\hline Cardenalli et al. (2019) & $\begin{array}{l}\text { A load-shedding framework, able to be fit in } \\
\text { runtime depending on the system workload is } \\
\text { proposed. }\end{array}$ & $\begin{array}{l}\text { Event retaining based on event metadata on the } \\
\text { data processor }\end{array}$ \\
\hline Slo et al. (2019) & $\begin{array}{l}\text { A load-shedding technique focused on the mutual } \\
\text { influence between events to determine its } \\
\text { importance }\end{array}$ & $\begin{array}{l}\text { A probabilistic model learning the event's } \\
\text { importance based on its relative position in } \\
\text { different windows and their types. }\end{array}$ \\
\hline Gupta and Hewett (2019) & $\begin{array}{l}\text { An adaptive normalization of data streams without } \\
\text { requiring indicating a specific application } \\
\text { previously. }\end{array}$ & $\begin{array}{l}\text { A distributed approach to adaptive normalization } \\
\text { of data streams }\end{array}$ \\
\hline Zhao et al. (2020) & $\begin{array}{l}\text { A load shedding technique complementing the } \\
\text { traditional approach on data items with } \\
\text { information about the event occurrences and its } \\
\text { impact before deciding about retaining or } \\
\text { discarding a given element. }\end{array}$ & $\begin{array}{l}\text { An analysis of each data contextualized by the } \\
\text { occurrences of other events jointly with its partial } \\
\text { or total impact on data items. }\end{array}$ \\
\hline Yan et al. (2018) & $\begin{array}{l}\text { Applying an incremental Kalman filter to } \\
\text { approach the current state }\end{array}$ & $\begin{array}{l}\text { The incremental algorithm is understood to be } \\
\text { applied in a multisensory environment to remove } \\
\text { systems errors, considering that context could } \\
\text { influence on sensors and their data. }\end{array}$ \\
\hline Watson et al. (2020) & $\begin{array}{l}\text { An Incremental State Estimation based on data } \\
\text { coming from sensors }\end{array}$ & $\begin{array}{l}\text { The covariance estimation is performed jointly } \\
\text { with } \mathrm{Z} \text { scores to determine when the data would } \\
\text { be considered or not to update a Gaussian Mixture } \\
\text { Model (GMM) }\end{array}$ \\
\hline Xu et al. (2019) & $\begin{array}{l}\text { A model of computation and transmission as a } \\
\text { tandem queue to analyze the information } \\
\text { freshness in an IoT network is proposed }\end{array}$ & $\begin{array}{l}\text { A joint analysis of the influence of data } \\
\text { preprocessing and data transmission tasks on the } \\
\text { information freshness. }\end{array}$ \\
\hline
\end{tabular}

In Gupta and Hewett (2019), authors describe an Apache Storm-based strategy to normalize data without being it associated with a specific application (e.g., a classification). This normalization strategy is introduced from a distributed approach to the adaptive normalization of data streams. As a similarity, the adaptive normalization is introduced in general terms and not specifically associated with a given application. As a difference, our proposal analyzes close to data sources each measure in terms of the metadata indicated in the project definition before normalizing them. In Zhao et al. (2020) the proposal is referred to complement the item's information with data about the event's states to analyze the pertinence or not of retaining certain data instead of analyzing data in isolation. As a similarity, the contextual analysis around the data is performed based on events to analyze them in a given context. As a difference, our proposal is focused on data collection systems positioned as close as data sources as possible, specialized in measurement projects.

In Yan et al. (2018), a proposal of the incremental Kalman filter is introduced to improve the accuracy associated with the determining of the state. This approach is addressed considering a 
International Journal of Mathematical, Engineering and Management Sciences

Vol. 6, No. 1, 363-382, 2021

https://doi.org/10.33889/IJMEMS.2021.6.1.023

multisensory environment and the mutual influence between sensors and context. As a similarity, an online filter-based technique is used to approach the current values of the metrics under monitoring to approximate the current state. As a difference, our proposal is based on a measurement framework that allows describing the entity's characterization before implementing a data stream monitoring. Thus, when data are missing or suspicious, metadata coming from the MandE project definition allows contrasting them to the expected variation range, unit, scale, among other aspects to collectively estimate the current scenario and associated entity state.

In Watson et al. (2020), an interesting proposal to approach the state estimation using a covariance adaptation is introduced. An incremental point of view to estimate the covariance while data come from sensors is described. As a similarity, an incremental covariance matrix is estimated to determine the presence of dependence or not among the involved metrics (or variables). As a difference, our focus on the covariance estimation is related to a load-shedding technique that acts collaboratively with GF to optimize resources and avoids making data transmissions that potentially could saturate the data processor.

In Xu et al. (2019), authors describe a strategy to regulate data transmissions based on an approach min-max programming. The idea is to minimize the maximum average of the peak age of information (PAoI) about updates related to data coming from sensors. Also, the authors describe an interesting perspective to analyze the data freshness in an IoT network based on the joint effects of certain data preprocessing tasks and data transmission.

\section{The Data Buffer and Incremental Estimating}

Using the measurement project definition at the startup, the MA reads from a single XML file the set of metrics to be implemented jointly with specifications about the devices. This file is organized following an extended measurement framework substantiated in a measurement ontology to early agree about each involved concept (e.g., what a metric, scale, unit, among other concepts mean). The schema based on such a measurement framework receives the name of CINCAMI/Project Definition (CINCAMI/PD) and it describes for a monitoring project, the entity under monitoring (e.g., an outpatient), the attributes to be monitored (e.g., the breath frequency), metrics responsible for quantifying each attribute (e.g., the value of the breath frequency, expressed in a given scale and unit where the normal expected range belongs to a given interval), the instrument to be used to get each measure based on a given metric (e.g., a sensor is assigned with this purpose), among other aspects related to how the measure is obtained and not about the measure itself. In other words, the CINCAMI/PD file is responsible for guiding every MA to know how a measure should be obtained using the related sensors and MA organizes the data buffer accordingly.

Thus, MA is responsible to pair the metric's definition (i.e., this is contained in the CINCAMI/PD file) with each associated device and create the data buffer. The data buffer will be dimensioned based on the number of metrics and the max number of measures to be simultaneously kept in memory. The maxMeasures parameter will guide the creation process to indicate the max capacity of the data buffer. Because it is an arbitrary parameter, it could be modified at any time depending on the hardware where the MA will run (e.g., an Arduino One, a Raspberry Pi 3, etc.).

It is important to highlight that the project definition is not a static concept, which means that it could change over time based on new requirements. In such a situation, this implies that DSPS could send a new definition file that regenerates dynamically in MA the data buffer according to the new definition. 
International Journal of Mathematical, Engineering and Management Sciences

Vol. 6, No. 1, 363-382, 2021

https://doi.org/10.33889/IJMEMS.2021.6.1.023

Figure 1 synthesizes the organization and global behavior of the data buffer. The data buffer in the MA is organized as a logical window with an arbitrary capacity that is defined using a parameter maxMeasures. Each time that sensors inform measures, they are added chronologically into the data buffer incorporating the timestamp and keeping the order given by the paired metrics (e.g., the position 0 corresponds to the value of the corporal temperature and so on). When the data buffer reaches the defined limit, the oldest data vector (i.e., the left extreme in Figure 1) will be discarded to be replaced by the newest (i.e., the right extreme in Figure 1). In this way, the data buffer seems to be unlimited, but indeed, it keeps the last measures related to sensors based on the value established for the maxMeasures parameter.

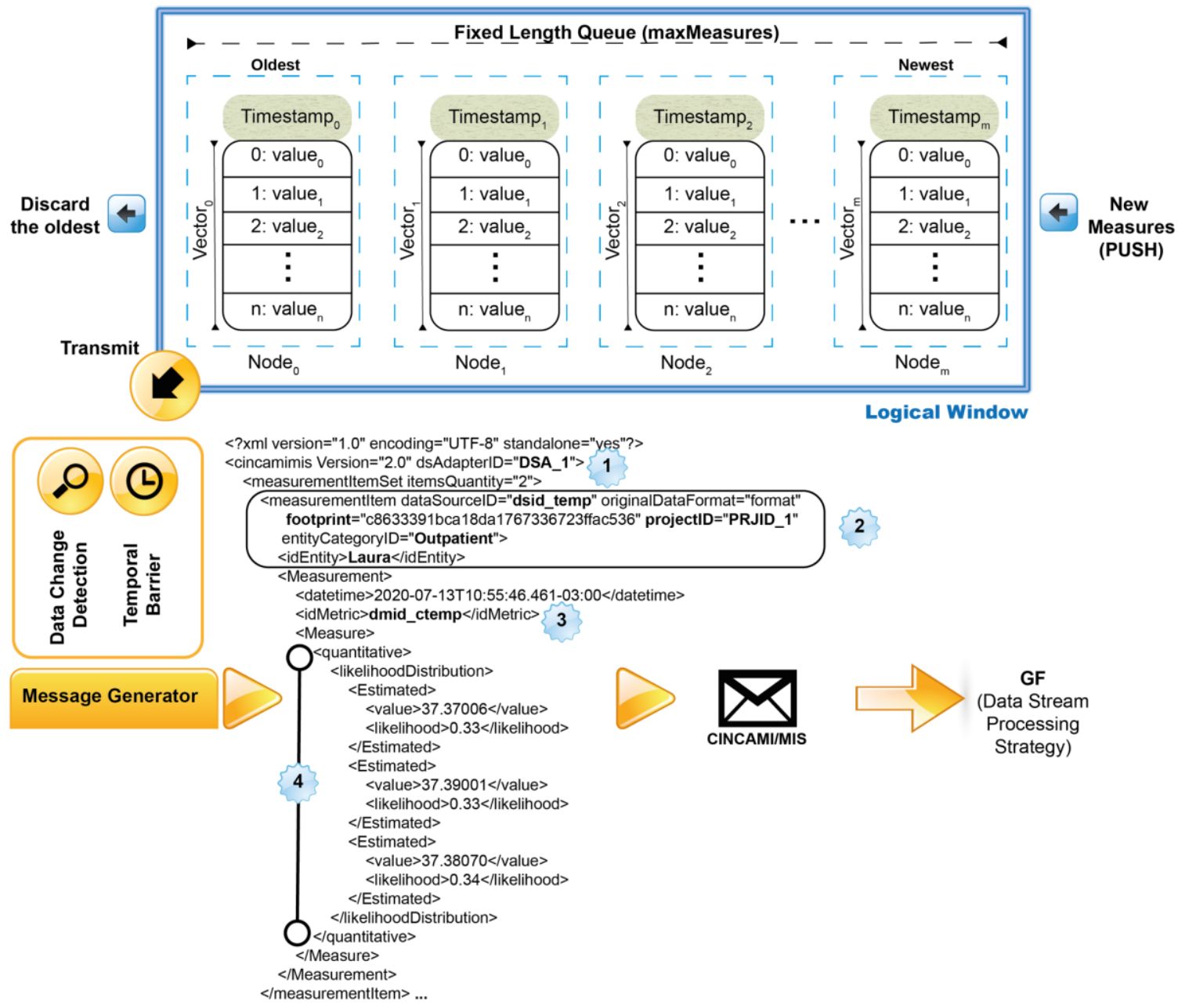

Figure 1. A conceptual perspective of the data buffer and the measurement interchange schema.

The data buffer keeps the last measures in memory waiting for a signal to transmit them. This signal could have an origin from two different points of view 1) A data change detection: each metric has an online filter implemented following the approach of (Cao and Rhinehart, 1997; Alford et al., 2018) which allows informing when there is enough proof that a data change has occurred, or 2) A temporal barrier: it establishes a minimum timespan through which at least one data transmission should be made. This is a complement to the data change detection because when no data change 
International Journal of Mathematical, Engineering and Management Sciences

Vol. 6, No. 1, 363-382, 2021

https://doi.org/10.33889/IJMEMS.2021.6.1.023

is detected for a given time, the temporal barrier will transmit the current data in the buffer to provide a proof-of-life. This articulation allows implementing a smart data transmission strategy, avoiding continuous data transmission, and fostering a rational use of resources.

When the transmission signal is originated from an active detector (no matter whether it was originated by the temporal barrier or a data change detection), the data buffer content is structured under the CINCAMI/Measurement Interchange Schema -CINCAMI/MIS-(Diván and Sánchez Reynoso, 2020b) to be transmitted immediately to the Gathering Function at DSPS. Each CINCAMI/MIS message is generated based on the CINCAMI/PD project definition which allowed structuring the data buffer. From the project definition, information about the entity ID, data source ID, metric ID, the kind of expected value (e.g., estimated or deterministic), among other aspects is obtained to be incorporated in the messages with measures. Because the project definition could consider complementary data (e.g., the geographic positioning related to a measure), each CINCAMI/MIS message could structurally vary according to whether it informs complementary data or not.

As it is possible to appreciate in Figure 1, each CINCAMIMIS message contains data (i.e., measures) but also metadata (i.e., tags) describing the meaning based on the measurement project definition. Each tag jointly with its corresponding IDs allows DSPS to interpret the measure contained in each CINCAMI/MIS message in its corresponding context. For example, the "dsAdapterID" tag indicates a specific MA acting as the message's origin jointly with the version of it (See the star with 1 in Figure 1). The rectangle associated with the star 2 indicates the ID for the origin's sensor (e.g., dsid_temp), a footprint to verify the measure content, the associated project ID (e.g., PRJID_1), and the associated entity category (e.g., Outpatient). The star with 3 in Figure 1 explicitly indicates the associated metric (e.g., dmid_ctemp) with the values specified in the star with 4 , which in the example are associated with a likelihood distribution (alternatively, it could be a single deterministic value too). For that reason, when GF receives a CINCAMIMIS message knows the way to process each value due to each ID allows looking up the associated definition for its interpreting.

$$
Z_{\text {score }}=\frac{x-\bar{x}}{s}
$$

As it is possible to appreciate in equation (1), to reach a Z-score, it is necessary to estimate at least the arithmetic mean and standard deviation (i.e., $\bar{x}$ and $\mathrm{s}$ respectively), but the data buffer is continuously receiving data from sensors while it discards the oldest ones. For such a reason, an incremental strategy to estimate each one is necessary.

$$
\bar{x}_{t}=\frac{\bar{x}_{t-1} * n_{t-1}+x_{t}}{n_{t-1}+1}
$$

Equation (2) provides a simple way to update continuously the arithmetic mean each time that a new measure arrives. It obtains the original sum through the product between the current arithmetic mean (i.e., $\bar{x}_{t-1}$ ) with the current number of processed measures (i.e., $n_{t-1}$ ). Such a product is added to the new received measure (i.e., $\bar{x}_{t}$ ) and the result is divided by $n_{t-1}$ plus one.

The standard deviation in a " $t$ " time is estimated through the sample variance using equation (3). In this sense, it is important to highlight that the estimation uses the current arithmetic mean 
International Journal of Mathematical, Engineering and Management Sciences

Vol. 6, No. 1, 363-382, 2021

https://doi.org/10.33889/IJMEMS.2021.6.1.023

available at the time in which the measure has arrived.

$$
s_{t}=\sqrt{\frac{\sum_{t=1}^{n}\left(x_{t}-\overline{x_{t}}\right)^{2}}{n-1}}
$$

Equation (4) schematizes this situation, where the arithmetic average for each time is not necessarily equal to the predecessor or successor due to the updating associated with each arrived value according to equation (2).

$$
s_{4}=\sqrt{\frac{\left(x_{1}-\bar{x}_{1}\right)^{2}+\left(x_{2}-\bar{x}_{2}\right)^{2}+\left(x_{3}-\bar{x}_{3}\right)^{2}+\left(x_{4}-\bar{x}_{4}\right)^{2}}{4-1}}
$$

The implementation of equations (3) and (4) require keeping in memory the accumulators jointly with the number of processed measures. However, it was said that the logical window will discard the oldest data vectors to retain the new ones, and such an action will affect equations (2), (3), and (4) because a part of the sum of equation (2) or the difference between the measure and its arithmetic average in equations (3) and (4) will be discarded. To avoid this situation, equations (5) and (6) are fitted as follows supposing a maxMeasures parameter with a value of 100.

$$
\bar{x}_{t}=\frac{\bar{x}_{t-1} * n_{t-1}+x_{t}-x_{t-100}}{n_{t-1}+1-1}
$$

Equation (5) rests the value to be discarded (i.e., $x_{t-100}$ ) and at the same time it discounts in 1 the divisor, while the new value is incorporated (i.e., $x_{t}$ ).

$$
s_{t}=\sqrt{\frac{\left(\sum_{t=1}^{n}\left(x_{t}-\bar{x}_{t}\right)^{2}\right)-\left(x_{t-100}-\bar{x}_{t-100}\right)^{2}}{n-1-1}}
$$

Something similar to equation (5) occurs in equation (6). The difference related to the oldest data vector to be discarded (i.e., $\left.\left(x_{t-100}-\bar{x}_{t-100}\right)\right)$ is decreased from the accumulator at the time the new difference is incorporated. The divisor is decreased in 1 but $\mathrm{n}$ is parallelly increased by the new measure, having as a limit the maxMeasures parameter (i.e., $n \leq$ maxMeasures). The implementation of equation (6) requires that for each data vector in memory, the estimated arithmetic average in a given instant be jointly stored to process the eventual discarding after.

Alternatively, equation (7) proposes an option to equation (6) to obtain incrementally the variance without needs to keep the accumulators in memory. This alternative requires to know the previous deviation estimation, the previous arithmetic average estimation, and the number of observations. However, even when they look similar, they have a conceptual difference. On the one hand, equation (6) goes progressively fitting its value according to the last "100" data vectors in consonance with the arithmetic average estimation (See equation (5)), allowing a better characterization of it. On the other hand, equation (7) goes estimating the variance from the beginning of the data series and not based on the last values.

$$
s_{t}=\sqrt{\frac{n}{n+1} *\left[s_{t-1}^{2}+\frac{\left(x_{t}-\bar{x}_{t-1}\right)^{2}}{n+1}\right]}
$$


International Journal of Mathematical, Engineering and Management Sciences

Vol. 6, No. 1, 363-382, 2021

https://doi.org/10.33889/IJMEMS.2021.6.1.023

Thus, equation (7) would be a better alternative to study the whole deviation estimation when it is required. However, equation (6) would be a better choice option when the partial sum needs to be used to estimate the covariances among metrics and the deviation should be explicative of the data contained in the data buffer as occurs here.

Let " $i$ " and " $j$ " two metrics to be implemented in a MA, the covariance between them could be calculated using equation (7) based on equation (6) as follows.

$$
\operatorname{Cov}(i, j)=\frac{\left[\sum\left(x_{i}-\bar{x}_{i}\right) *\left(x_{j}-\bar{x}_{j}\right)\right]-\left[\left(x_{i-100}-\bar{x}_{i-100}\right) *\left(x_{j-100}-\bar{x}_{j-100}\right)\right]}{n-1-1}
$$

Equation (8) would require keeping in memory the differences to subtract them when the data vector is discarded. Thus, given a number $m$ of metrics and utilizing equation (8), it is possible to approach the covariance matrix incrementally according to the logical window as it is indicated in equation (9).

$$
\operatorname{Cov}=\left[\begin{array}{ccc}
s^{2}{ }_{1} & \ldots & \operatorname{Cov}_{1 m} \\
\ldots & \ldots & \ldots \\
\operatorname{Cov}_{m 1} & \ldots & s^{2}{ }_{m}
\end{array}\right]
$$

The covariance matrix described by equation (9) is triangular because $\operatorname{Cov}(i, j)=\operatorname{Cov}(j, i)$. This allows implementing as a unidimensional array mapping the elements according to a triangular matrix, optimizing the use of memory. Also, from equation (8), it is possible to get Pearson's correlation matrix as it is indicated in equation (10).

$$
\left\{\begin{array}{l}
\forall i \neq j: r_{i, j}=\frac{\operatorname{Cov}(i, j)}{\sqrt{\operatorname{Cov}(i, i)} * \sqrt{\operatorname{Cov}(j, j)}}=\frac{\operatorname{Cov}(i, j)}{s_{i} * s_{j}} \\
\forall i=j: r_{i, j}=\frac{\operatorname{Cov}(i, j)}{\sqrt{\operatorname{Cov}(i, i)} * \sqrt{\operatorname{Cov}(j, j)}}=\frac{s^{2}{ }_{i}}{s_{i} * s_{i}}=1
\end{array}\right.
$$

The library pabmmCommons library is available on GitHub (https://github.com/mjdivan/pabmmCommons) and it incorporates the object model described by Figure 2 jointly with the implementation of the mentioned formulas and data buffer for the realtime data processing. The library is written in Java 8 and it was released under the terms of the Apache 2.0 license.

Figure 2 highlights with yellow background the new classes incorporated in the pabmmCommons library. The SPCMeansBasedNode class is a specialization of the SPCBasedBufferNode class with the possibility to keep the estimated arithmetic averages and differences with the measures concurrently in memory under the same node into the data buffer.

The SPCMeansBasedBuffer class implements incremental computing of arithmetic averages and variances when the data vector with measures is incorporated. This class is a specialization of SPCBasedBuffer class that keeps in memory a logical window implemented through a concurrent linked queue of SPCMeansBasedNode instances. The class implements the Observer interface to monitor the online data change detectors and know when a data change has occurred. Also, it extends the Observable class to inform to the message generator (See Figure 1) that a new data 
International Journal of Mathematical, Engineering and Management Sciences

Vol. 6, No. 1, 363-382, 2021

https://doi.org/10.33889/IJMEMS.2021.6.1.023

transmission could be carried forward, be it as a consequence of a temporal barrier or the data change detectors.

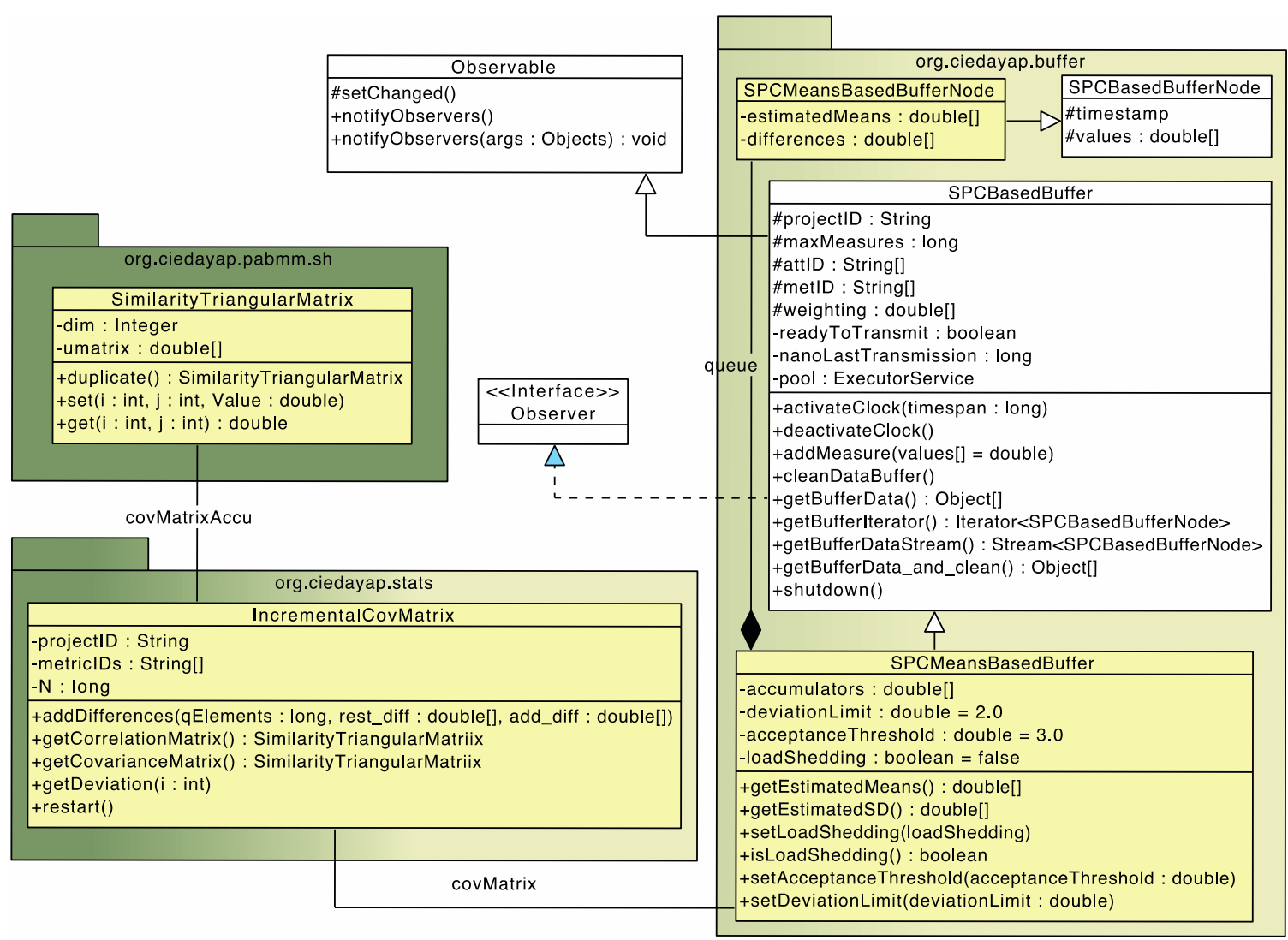

Figure 2. Main classes related to the data buffer with the capacity of estimating incrementally.

The SimilarityTriangularMatrix class implements a triangular matrix through the mapping from/to a unidimensional array. The IncrementalCovMatrix class implements the computing for the incremental covariance and correlation matrixes, using an instance of the SimilarityTriangularMatrix class to manage data in memory. When a data transmission is performed, the data buffer is cleaned, and all the estimations will be restarted again.

The attributes deviationLimit, acceptanceThreshold, and loadShedding are directly associated with the application of the load-shedding technique that is detailed then.

\section{A Metadata and Z Score-based Load-Shedding Technique}

From the estimation of the arithmetic averages and standard deviations for each metric, it is possible to get the Z-scores which are especially useful to avoid the effect of variables with great magnitude in detriment of those with a different variation range. The set of variables (or metrics) managed to a measurement adapter is established in the measurement project definition, which is paired with sensors during the startup. The metadata is associated with the data buffer, where it is possible to 
International Journal of Mathematical, Engineering and Management Sciences

Vol. 6, No. 1, 363-382, 2021

https://doi.org/10.33889/IJMEMS.2021.6.1.023

appreciate the set of metrics managed (See the metID attribute in the SPCBasedBuffer class) jointly with its weightings (See the weighting attribute in the SPCBasedBuffer class). The weighting expresses the relative importance of each metric in the measurement project and it is defined by the project director. When the loadShedding attribute in the SPCMeansBasedBuffer class is established to false (it is the default value), the data buffer will transmit data following two complementary policies. On the one hand, when an online filter informs that a data change has occurred. On the other hand, when a temporal barrier indicates that transmission must be done as a proof-of-life because it has elapsed a certain time without any transmission. Each time that an online filter is triggered indicating a data change, the temporal barrier is restarted to zero. The last one only is triggered when there not exists a data transmission after a certain time (15 seconds by default).

Previously, DSPS only applied the load-shedding technique in GF but when it was applied, the data have already been transmitted and they were discarded in GF. That is to say, GF is the architectural receiver of all measures coming from a certain number of heterogeneous measurement adapters. All of them are physically located at different points with autonomous behavior. For example, Figure 3 conceptually describes two different measurement projects. On the one hand, project 1 is related to the outpatient monitoring and each MA is related to a given outpatient under monitoring. On the other hand, project 2 refers to a greenhouse monitoring where MA2 and MA"n" monitor different locations. In this sense, some considerations are worthy to be highlighted 1) Project 1 and 2 are initialized using the corresponding project definition through the CINCAMI/PD message, 2) The CINCAMIPD message between project 1 and 2 are structurally different because their aims are completely different, 3) The CINCAMI/MIS related to project 1 and 2 are structurally different. In the same project, the informed measures from measurement adapters will be conceptually different because they are monitoring different entities (though structurally similar), 4) GF takes knowledge from collected measures once each CINCAMI/MIS message has been transmitted through the Internet using the Cloud and not before, 5) GF receives simultaneously CINCAMIMIS messages from different MA associated with different projects, 6) From the MA perspective, the processing rate of GF is unknown, and 7) GF could send a message to each MA but not manage its data processing.

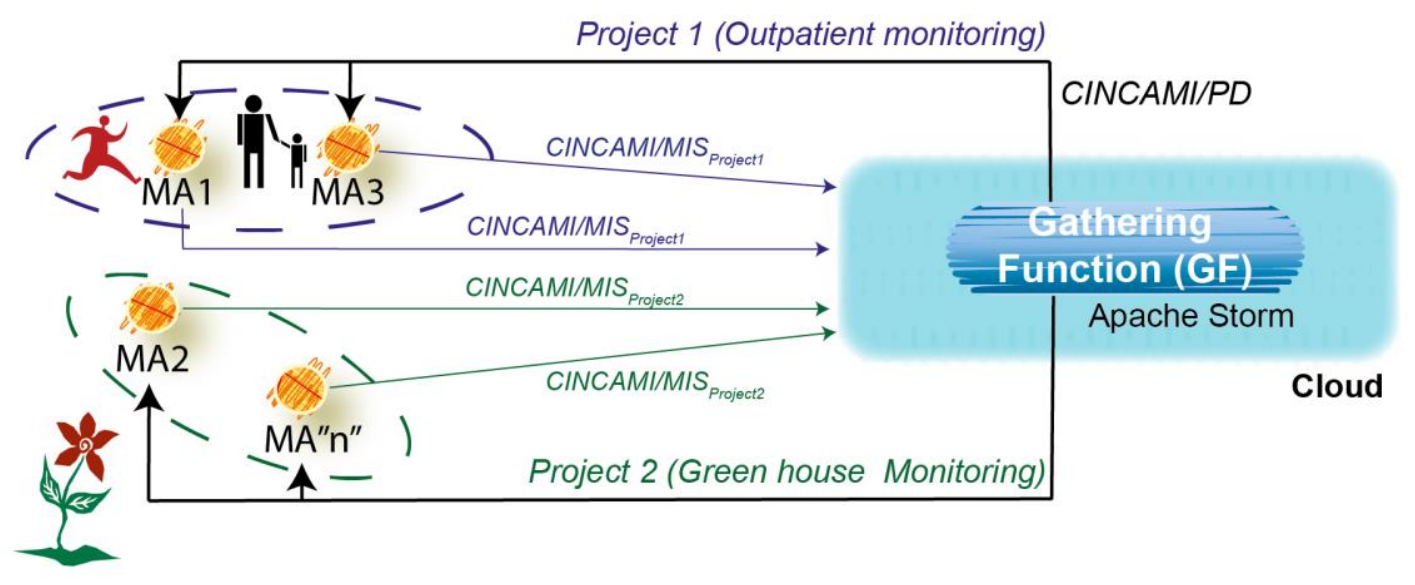

Figure 3. Conceptual description of the location of GF and MA and its impact on the data transmission.

Thus, the idea is to complement to GF with a local load shedding implemented on the MA before the data transmission is performed. Thus, when GF is close to reaching a risky processing rate 
International Journal of Mathematical, Engineering and Management Sciences

Vol. 6, No. 1, 363-382, 2021

https://doi.org/10.33889/IJMEMS.2021.6.1.023

according to (Diván and Sánchez Reynoso, 2020a), it could request to MA that the local loadshedding techniques need to be activated through the activation of the loadShedding flag from the SPCMeansBasedBuffer class. This will imply that MA will filter the data to be retained in the buffer using equation (11), while only will perform data transmissions when a data change is detected, discarding any intent of data transmission as a proof-of-life.

$$
\sum_{i=1}^{m} \vec{z}_{x_{i}} * \vec{w}_{i}<\text { acceptanceThreshold }
$$

Equation (11) describes the data retention policy of each MA based on an acceptanceThreshold parameter which is indicated in each project definition. The vector $\vec{z}_{x_{i}}$ is the Z-score vector computed from the estimation of the arithmetic average and the deviation for each metric. The vector $\vec{w}_{i}$ contains the weighting of each metric defined in the project definition to know the relative importance of each one. Thus, the joint measures from sensors will be retained in the data buffer when the product does not exceed the established threshold, otherwise, the measures are discarded because the focus is applied to the general behavior and not in outliers throughout the period where the load-shedding technique is enabled.

Thus, when a data vector is received, it is standardized using the estimations for the average and standard deviation. When the Z-score of a variable individually exceeds the deviation limit (See the deviationLimit attribute in the SPCMeansBasedBuffer class, it has a value of 2 by default), it will be accumulated multiplying it by its corresponding weighting. In the end, the data vector will be retained in the data buffer when the cumulated sum of the product between Z-scores and weightings do not exceed the acceptance threshold (See the acceptanceThreshold attribute in the SPCMeansBasedBuffer class, it has a value of 3 by default).

This kind of filtering strategy is dynamical because the estimations go updating continuously on each new data vector, and the fact of discard an excessive variation is related to study the general behavior instead of the outlier itself. The metric's metadata allows knowing the relative importance of each variable in the project to decide to retain a given data vector.

In this way, only the data following the general behavior about updated estimators are retained in the data buffer, while the message generator will inform data from MA only when a data change has occurred. An analysis of discrete simulations based on the number of data transmissions and associated resources with the data buffer are outlined in the next sections to provide some initial applicability patterns.

The possibility to incorporate the covariance and correlation matrix allows detecting dependencies between variables, leaving as a future work other kinds of dependences analysis jointly with its impact on the acceptance/rejection policies related to online filters.

\section{Discrete Simulations on the Data Buffer: Methodological and Quantitative Points of View}

Four discrete simulations were scheduled to provide initial applicability patterns about the proposed updates in the pabmmCommons library. All simulations have a common region related to the initialization of the Java Instrumentation Agent to measure the runtime memory consumption and the initialization of a numbers' random generator jointly with the loading of a measurement project 
International Journal of Mathematical, Engineering and Management Sciences

Vol. 6, No. 1, 363-382, 2021

https://doi.org/10.33889/IJMEMS.2021.6.1.023

containing 10 metrics. The metrics' information based on whether the load-shedding technique is activated or not will derivate in a given initialization of the data buffer according to the chosen simulation.

All simulations were performed on a MacBook Pro running macOS Catalina 10.15.5 with a 2,9 GHz Intel Core i7 processor, 16GB $2133 \mathrm{MHz}$ LPDDR3 of RAM, and a Radeon Pro 560 4GB of dedicated RAM. It was used Netbeans 9 on Java 8 (A runtime environment version 1.8.0_191) on a 64-bit platform. A BPMN diagram detailing the simulations' steps is synthesized in Figure 4.

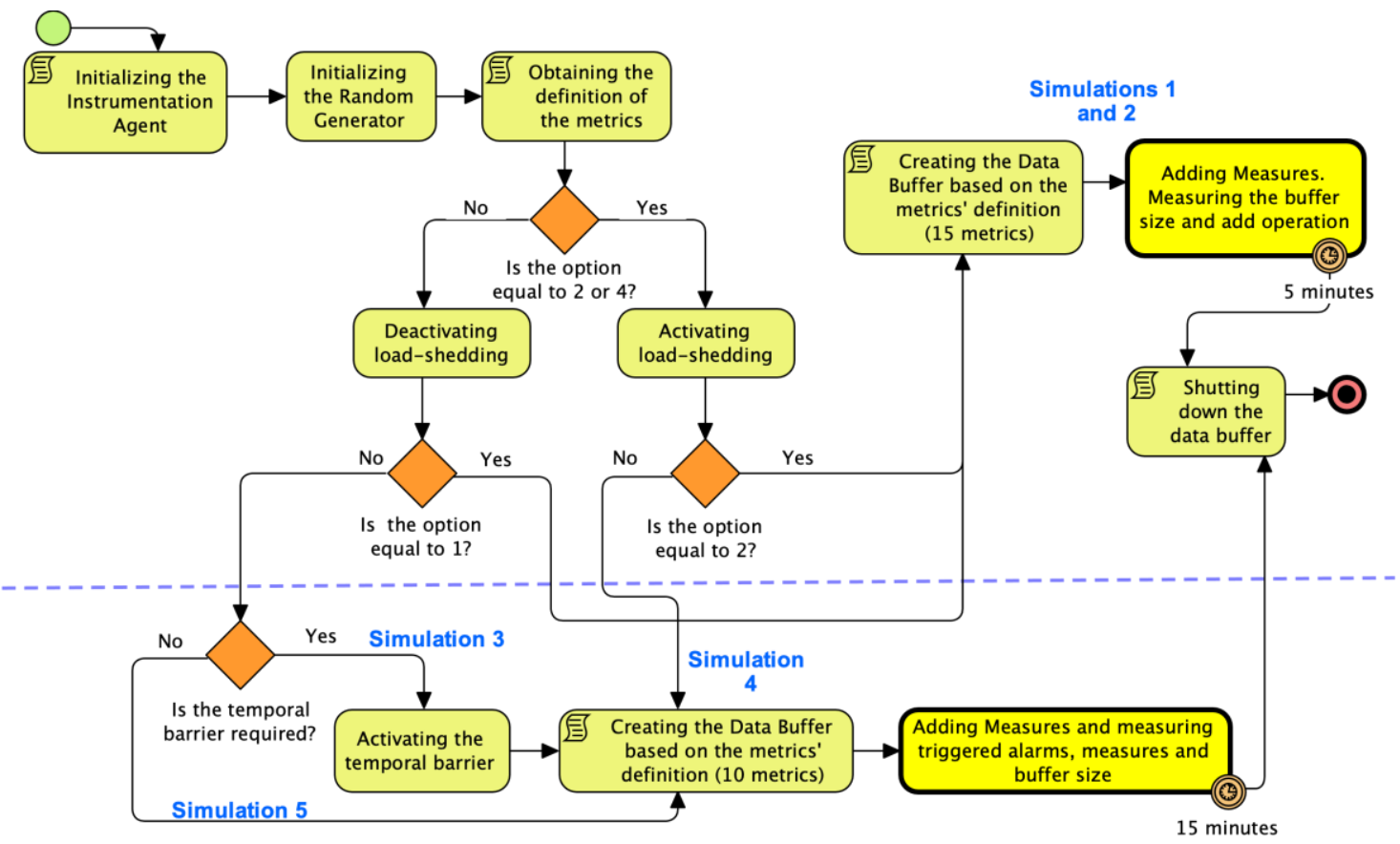

Figure 4. A BPMN diagram describing the discrete simulation methodology.

Simulations 1 and 2 were oriented to measure the add operation in the data buffer when the loadshedding technique is enabled (i.e., the simulation 2), or when it is disabled (i.e., the simulation 1). Both simulations ran for 5 minutes, incorporating each $100 \mathrm{~ms}$ a data vector randomly generated (With an arithmetic average of 35 and a standard deviation of 2) in the data buffer. The vector dimensionality was associated with 15 metrics, while the data buffer was defined to retain 1000 data vectors. Once the data vector had been incorporated, measures about the required time and the current size of the data buffer are updated through the console.

Simulations 3, 4 and 5 were oriented to analyze the behavior of the triggered alarms for data transmission from the data buffer. Simulation 3 describes the behavior when the load-shedding technique is disabled, making the data transmissions based on the online filter and temporal barrier (i.e., as a proof-of-life). Simulation 4 details the behavior with the load-shedding technique enabled and deactivating the temporal barrier. When the load-shedding is enabled the only considered alarm to perform a data transmission is based on the online filter detecting data changes, for such a reason the simulation time was increased up to 15 minutes monitoring concurrently 10 metrics, keeping the logical window with a capacity of retaining the last 1000 data vectors. Simulation 5 exposes a 
International Journal of Mathematical, Engineering and Management Sciences

Vol. 6, No. 1, 363-382, 2021

https://doi.org/10.33889/IJMEMS.2021.6.1.023

scenario where the load-shedding technique is disabled jointly with the temporal barrier, the transmissions are performed based on the online filter considering all data (i.e., no data discarding is allowed), while the estimation of arithmetic means and deviations of metrics is performed online using an instance of the SPCMeansBasedBuffer class.

As it is shown in Figure 4, once the simulations 1, 2, 3, or 4 reach the end, the data buffer is shut down, releasing the reserved resources, and ending the program. The implementations of these discrete simulations are available in the mainMeansBufferDetectors class in the org.ciedayap. simula package.

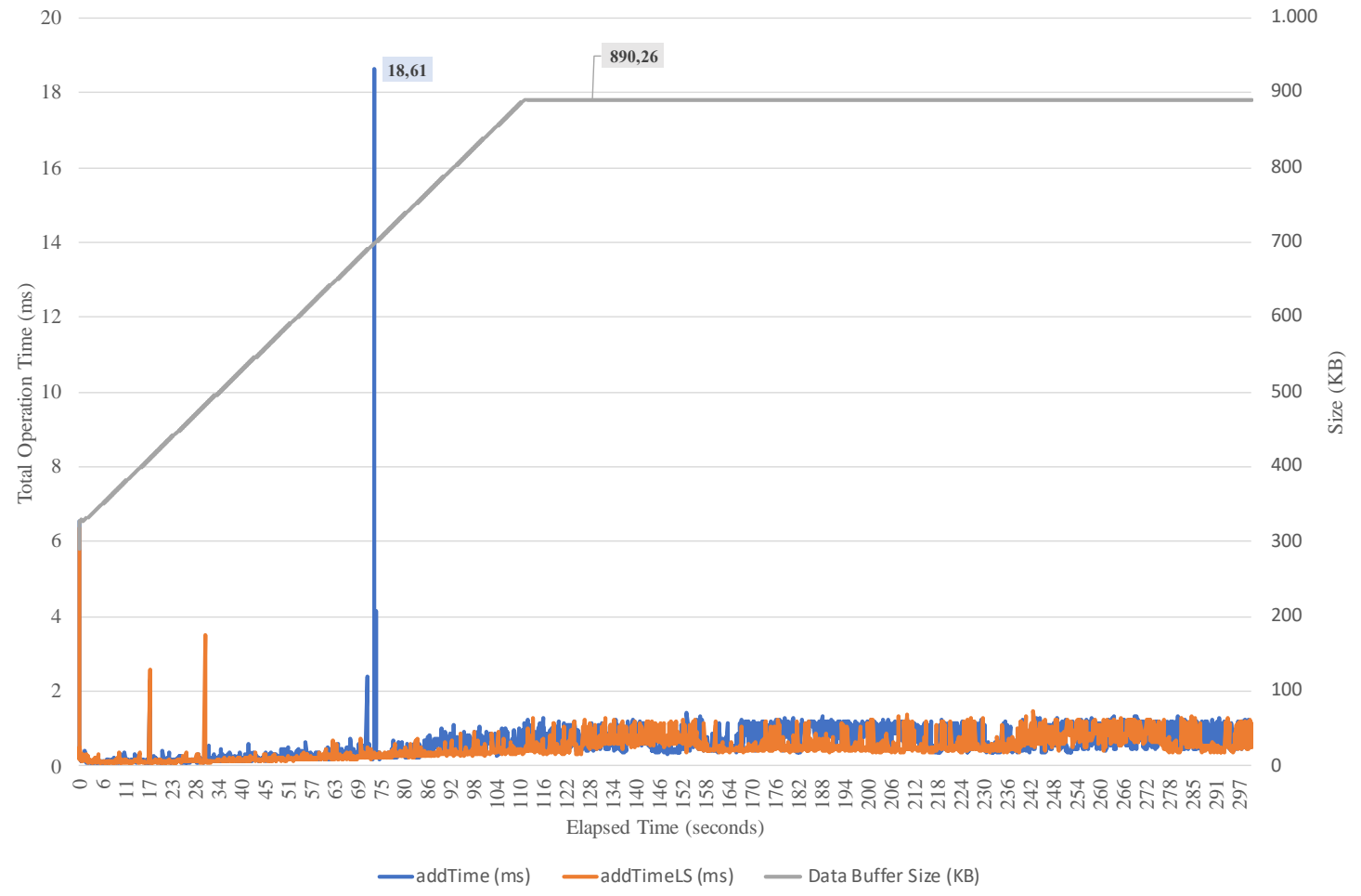

Figure 5. Evolution of the operation time and data buffer size throughout 5 minutes (Simulations 1 and 2).

Figure 5 shows the evolution of the "add" operation time jointly with the data buffer size when the load-shedding option is enabled (i.e., the simulation 2 which is identified with orange color), and when it is disabled (i.e., the simulation 1 which is identified with the blue color). The operation time is quantified using the left ordinate axis, while the size expressed in Kilobytes is represented on the right ordinate axis of the figure. The outliers are a consequence of Java's garbage collector that was operating while the simulation was performed. Independently of them, it is possible to appreciate that the "add" operation has a similar behavior though some differences could be highlighted in the boxplot in Figure 6. When the Load-Shedding (LS) technique was enabled, the "add" operation time through the data discarding was subtle better, reaching an arithmetic average of $0.49 \mathrm{~ms}$ and a median of 0.44 versus $0.62 \mathrm{~ms}$ and $0.54 \mathrm{~ms}$ when the LS was disabled. Also, the variation range was regulated when LS was enabled as it is possible to appreciate through the boxplot in Figure 6, which is consistent with their standard deviations obtained (i.e., 0.51 when LS 
International Journal of Mathematical, Engineering and Management Sciences

Vol. 6, No. 1, 363-382, 2021

https://doi.org/10.33889/IJMEMS.2021.6.1.023

is enabled versus 0.31 when it is disabled). Thus, the use of LS technique decreases in $0.1 \mathrm{~ms}$ the individual operation time considering its median, which represents an initial optimization of $22.72 \%$ (i.e., [[0.54/0.44]-1]*100).

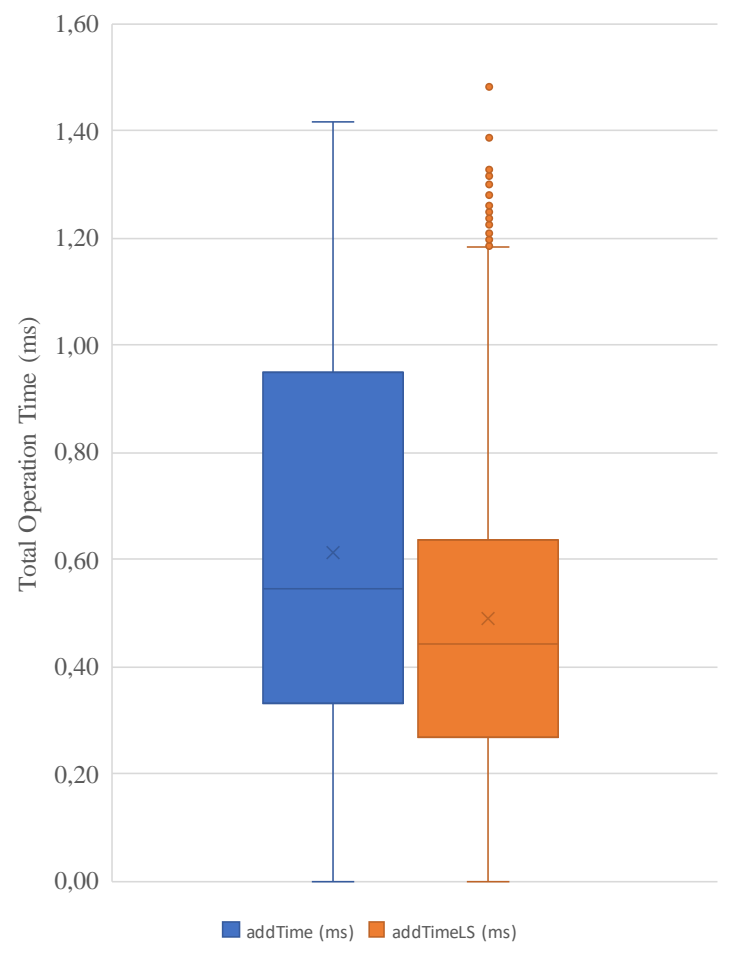

Figure 6. Boxplot for the addTime operation (without and with the Load-shedding enabled) throughout 5 minutes considering the measures under $2 \mathrm{~ms}$ for better visibility (Simulations 1 and 2).

Also, even when the LS technique is running in parallel, the logical window allows keeping stable the data buffer size, which can be appreciated in Figure 5. Once the logical window reaches the max occupation (i.e., 1000 data vectors in these simulations), it starts to discard the oldest data vectors to retain the new ones, keeping stable the data buffer size in $890,26 \mathrm{~Kb}$ for 10 metrics. The possibility of articulating the LS technique with a logical window in the data buffer allows retaining data that vary according to the most important metrics indicated in the project definition, avoiding an overhead in the "add" operation time.

However, the outlier introduces a temporal limitation about the applicability of the LS technique in conjunction with the data buffer based on a logical window. These incremental estimations are possible if and only if the time between data arriving is upper than $18,61 \mathrm{~ms}$. In the best case and scenario, this operation time could be reduced to $0.08 \mathrm{~ms}$. Also, it is important to indicate that a data buffer monitoring concurrently 10 metrics with 1000 available slots (i.e., up to 10,000 measures) would require $890,26 \mathrm{~KB}$ to store all information related to the data buffer and LS techniques.

Figure 7 describes the shown behavior by the number of triggered alarms and the volume of data transmissions throughout 15 minutes of simulation with the load-shedding technique disabled. The 
International Journal of Mathematical, Engineering and Management Sciences

Vol. 6, No. 1, 363-382, 2021

https://doi.org/10.33889/IJMEMS.2021.6.1.023

left ordinate axis indicates the number of triggered alarms, while the right ordinate axis indicates the number of slots (i.e., each slot contains a full data vector with all its variables) transmitted as a consequence of the triggered alarm. As it is possible to appreciate, the alarms triggered due to a temporal barrier are indicated with the blue color, while those related to a data change are represented with the orange color. Numbers over the bar describe the accumulated number of triggered alarms of such a type, for example, the last bar on the right side indicates 51 alarms with an origin in the temporal barrier and 8 related to a data change. That is to say, from 59 alarms in the 15 minutes, $86,44 \%$ corresponds to a temporal barrier in which it aims to provide a proof-oflife to GF. Just $13,56 \%$ of the data transmission is associated with data change.

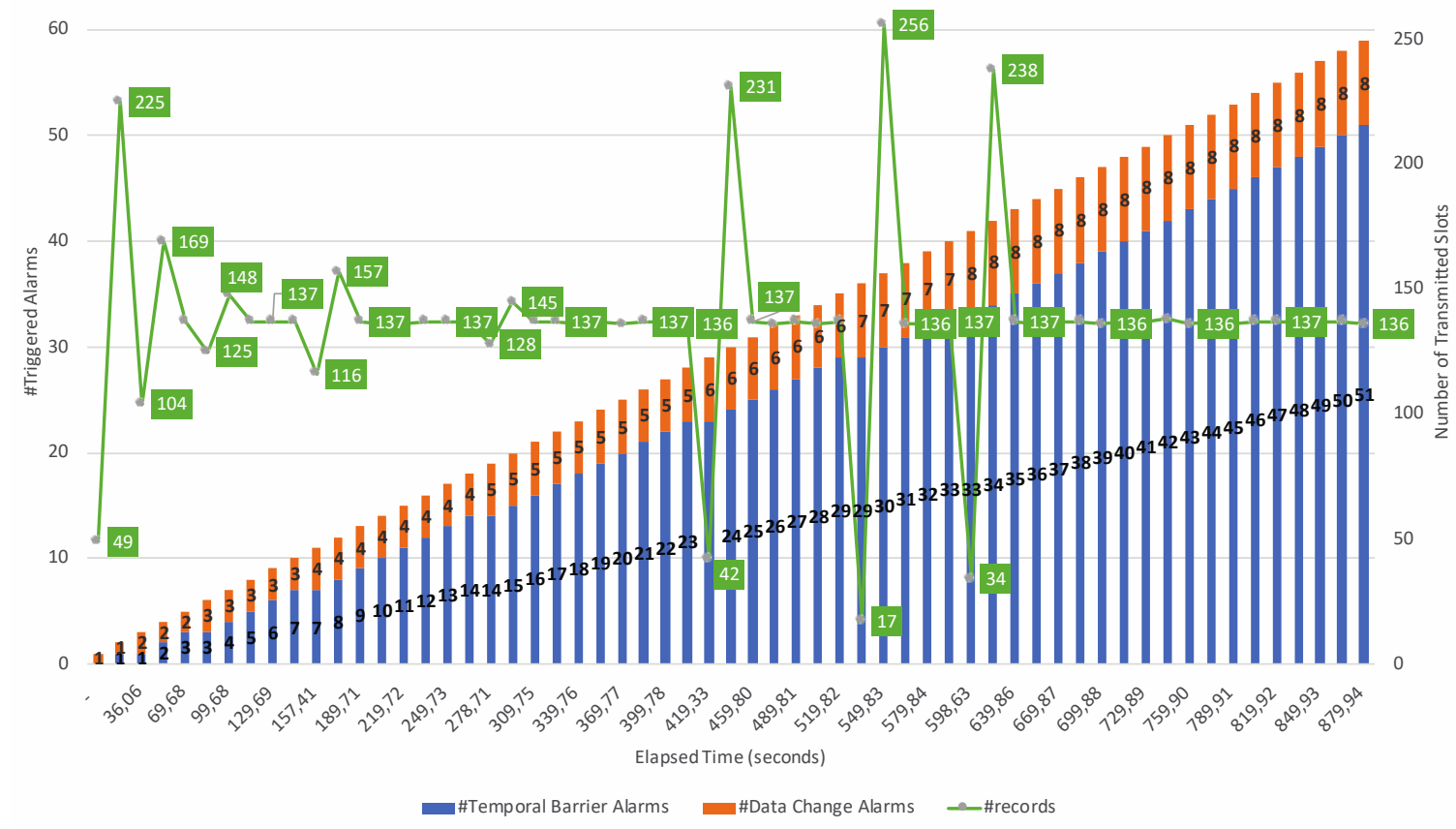

Figure 7. Evolution of the triggered alarms and data transmissions throughout 15 minutes (simulation 3 ).

Independently of the data transmission origin, an average of 136 slots are informed in each operation which represents around 1360 measures (i.e., 10 metrics $* 136$ measure/metrics). The minimum volume of informed data was 17 slots (i.e., 170 measures) consuming 14,26 KB, while the maximum volume was 256 slots (i.e., 2560 measures) consuming 117,88 KB.

Figure 8 describes the exposed behavior throughout 15 minutes of simulation with the loadshedding technique enabled. The left ordinate axis indicates the number of triggered alarms, while the right ordinate axis indicates the number of slots (i.e., each slot contains a full data vector with all its variables) transmitted as a consequence of the triggered alarm. Results seem to be promising because just 15 data transmissions were required for 15 minutes versus the 59 required without the technique. This avoids around $74.57 \%$ of data transmissions, consuming the resources associated with $25.43 \%$ of the original transmissions. Also, the volume of informed data has changed. When the technique is activated, the maximum number of transmitted data was 999 slots (i.e., 9990 measures) consuming $440.05 \mathrm{~KB}$, while the minimum number was 44 slots (i.e., 440 measures) consuming $25,96 \mathrm{~KB}$. As it was introduced, all the alarms in this simulation are associated with 
International Journal of Mathematical, Engineering and Management Sciences

Vol. 6, No. 1, 363-382, 2021

https://doi.org/10.33889/IJMEMS.2021.6.1.023

data changes detected through the online filters based on data that were retained accordingly with the Z-scores and the weightings of the defined metrics. Any kind of temporal barrier is deactivated when the load-shedding technique is enabled.

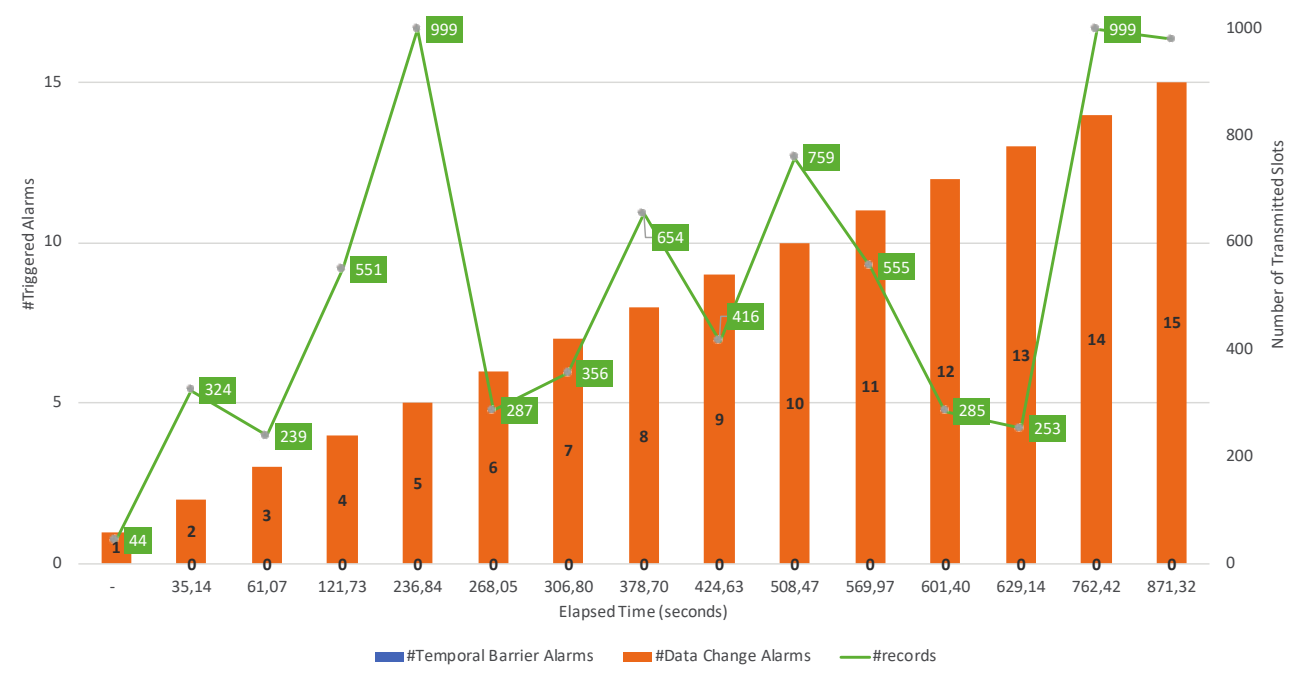

Figure 8. Evolution of the triggered alarms and data transmissions throughout 15 minutes with loadshedding technique enabled (simulation 4).

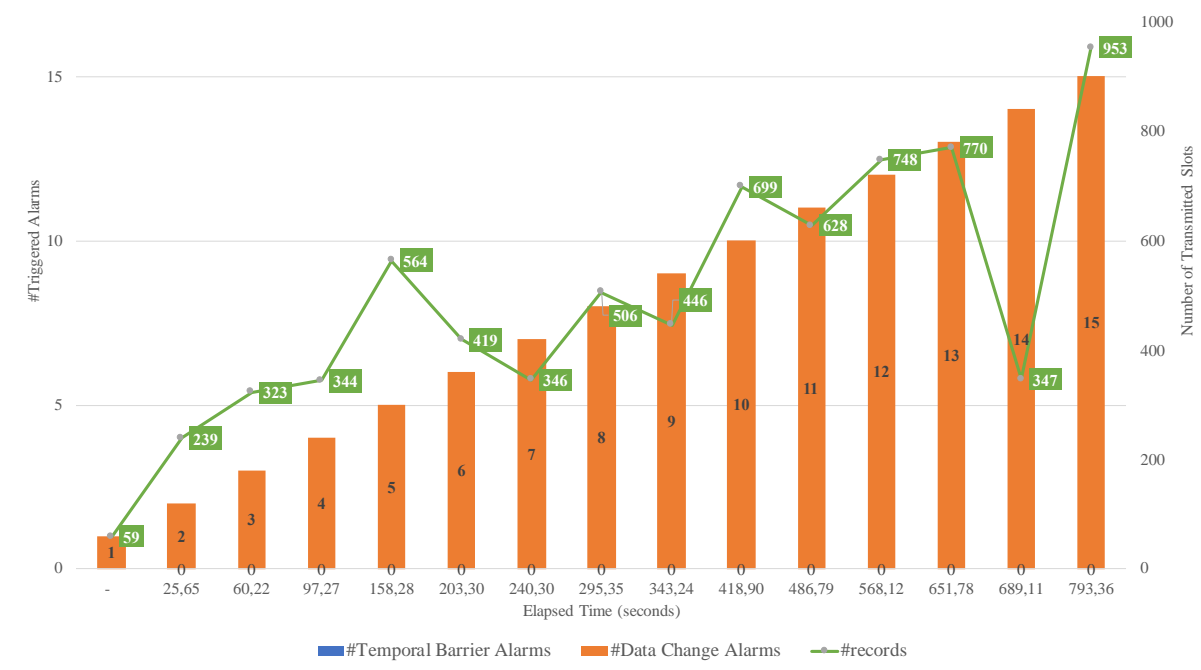

Figure 9. Evolution of the triggered alarms and data transmissions with the load-shedding technique and temporal barrier disabled (Simulation 5). 
International Journal of Mathematical, Engineering and Management Sciences

Vol. 6, No. 1, 363-382, 2021

https://doi.org/10.33889/IJMEMS.2021.6.1.023

Figure 9 describes the exposed behavior throughout 15 minutes of simulation with the loadshedding technique and proof-of-life disabled but computing the estimation for arithmetic average and deviations. The online filter is used to detect data changes and transmit accordingly. Analogously with Figure 8, the left ordinate axis indicates the number of triggered alarms, while the right ordinate axis indicates the number of slots (i.e., each slot contains a full data vector with all its variables) transmitted as a consequence of the triggered alarm. Comparing Figures 8 and 9, it is possible to appreciate that even when the number of data transmissions is the same, the loadshedding technique (See Figure 8) incorporates a delay to transmit. That is to say, reading from the second transmission's time onward in both figures, it is possible to appreciate a subtle delay when load-shedding is enabled, for example, the transmission 2 in Figure 9 occurred at 25,65 seconds while in Figure 8 at 35,14 seconds, the transmission 10 in Figure 9 was made at 418,9 seconds while in Figure 8 at 508,47 seconds, and in this way so on. The average time between transmissions is 57,67 seconds with the load-shedding disabled with a median of 446 slots by transmission, while it is 62,24 seconds with a median of 416 slots by transmission when the technique is enabled. For that reason, it would be probable that the number of transmissions with load-shedding disabled is higher when the considered period is higher than 15 minutes.

However, this result is interesting because if for any reason the load-shedding do not want to be used (e.g., to avoid discarding data), data transmissions limited to proof-of-life allow complementing to GF in front of an eventual overhead with similar results, keeping active the online estimation of the arithmetic averages and deviations.

\section{Conclusions}

This work has introduced a Metadata and Z-score based load-shedding technique in the measurement adapter articulated with a data buffer that implements a logical window behavior in the DSPS. The capacity to retain a given volume of data avoiding an overflow allows waiting for the precise instant to perform data transmission. Also, while the data are incorporated in the data buffer, an incremental strategy to estimate the arithmetic average, standard deviations, covariance, and correlations were introduced. Thus, such estimations were used to implement the data retention policy that is supported by the weightings coming from the measurement project to discriminate among different levels of importance for the metrics. Discrete simulations indicated that a minimum of $18.61 \mathrm{~ms}$ between data arriving from sensors is necessary to process the data in the buffer, while $890,26 \mathrm{~KB}$ was required to implement it.

It is worthy to mention the saving of resources generated by the load-shedding technique. In a normal situation, MA will transmit around 59 times ( 51 emerged from temporal barriers and 8 from data change detections) with 136 slots as average in each transmitted message. In contrast with it, with the load-shedding technique activated, MA only required 15 data transmissions increasing to 513 slots the average of data to be transmitted by each message. This represented a saving of around $74 \%$ in comparison to the original data transmissions, which is very interesting not only for the MA but also for the GF because now it is possible to request collaboration to MA to discard data before they are sent. That is to say, when the processing rate of GF is close to being exceeded, it could activate its global load-shedding technique and also it notifies to each MA to activate the local LS. Thus, this distributed behavior could decrease the data volume received by GF up to 74\%, allowing release resources and to solve the potential bottleneck in the DSPS. Simulation 5 showed an interesting alternative when no data want to be discarded. The alternative uses the online data filter to regulate the data transmissions, avoiding the temporal barriers, and computing in real-time the estimations about arithmetic averages and deviations, reaching similar results to the use of the load- 
International Journal of Mathematical, Engineering and Management Sciences

Vol. 6, No. 1, 363-382, 2021

https://doi.org/10.33889/IJMEMS.2021.6.1.023

shedding technique. However, the time between transmissions is lower than when the load shedding is used.

Thus, this approach would be feasible to be applied on a given device if device were able to manage a volume of $2 \mathrm{MB}$ of RAM (i.e., $1 \mathrm{MB}$ for the data buffer and load-shedding technique, while another equal is necessary to prepare the data message to be transmitted) it is warrantied a lifespan of at least $19 \mathrm{~ms}$ between each arrived data.

All the source code related to the pabmmCommons library for the extensions detailed jointly with the corresponding simulations here described are freely available on GitHub for your reference.

As future work, other kinds of dependencies analysis jointly with its impact on the data acceptance/rejection policies related to online filters will be analyzed.

\section{Conflict of Interest}

The authors confirm that there is no conflict of interest to declare for this publication.

\section{Acknowledgment}

This research is partially supported by the project CD 312/18 from the Economy School at the National University of La Pampa.

\section{References}

Alford, J.S., Hrankowsky, B.M., \& Rhinehart, R.R. (2018). Data filtering in process automation systems. InTech, 65(4), 14-19.

Cao, S., \& Rhinehart, R.R. (1997). A self-tuning filter. Journal of Process Control, 7(2), 139-148.

Cardellini, V., Mencagli, G., Talia, D., \& Torquati, M. (2019). New landscapes of the data stream processing in the era of fog computing. Future Generation Computer Systems, 99, 646-650.

Chen, Y., Li, M., Chen, P., \& Xia, S. (2019). Survey of cross-technology communication for IoT heterogeneous devices. IET Communications, 13(12), 1709-1720.

Diván, M.J., \& Sánchez Reynoso, M.L. (2020a). A load-shedding technique based on the measurement project definition. In: Jain, V., Patnaik, S., Popențiu Vlădicescu, F., Sethi, I. (eds) Recent Trends in Intelligent Computing, Communication and Devices. Springer, Singapore, 1006, pp. 1027-1033.

Diván, M.J., \& Sánchez Reynoso, M.L. (2020b). An architecture for the real-time data stream monitoring in IoT. In: Tanwar, S., Tyagi, S., Kumar, N. (eds) Multimedia Big Data Computing for IoT Applications. Springer, Singapore, 163, pp. 59-100.

Fernández-Rodríguez, J.Y., Álvarez-García, J.A., Fisteus, J.A., Luaces, M.R., \& Magaña, V.C. (2017). Benchmarking real-time vehicle data streaming models for a smart city. Information Systems, 72, 62-76.

Ghobaei-Arani, M., Souri, A., \& Rahmanian, A.A. (2020). Resource management approaches in fog computing: A comprehensive review. Journal of Grid Computing, 18, 1-42. 
International Journal of Mathematical, Engineering and Management Sciences

Vol. 6, No. 1, 363-382, 2021

https://doi.org/10.33889/IJMEMS.2021.6.1.023

Gupta, V., \& Hewett, R. (2019, November). Adaptive normalization in streaming data. In Proceedings of the 2019 3rd International Conference on Big Data Research (pp. 12-17). ACM. Cergy-Pontoise, France.

Izadpanah, R., Allan, B.A., Dechev, D., \& Brandt, J. (2019). Production application performance data streaming for system monitoring. ACM Transactions on Modeling and Performance Evaluation of Computing Systems, 4(2), 1-25.

Nour, B., Sharif, K., Li, F., Biswas, S., Moungla, H., Guizani, M., \& Wang, Y. (2019). A survey of internet of things communication using ICN: A use case perspective. Computer Communications, 142-143, 95-123.

Slo, A., Bhowmik, S., \& Rothermel, K. (2019, December). ESPICE: Probabilistic load shedding from input event streams in complex event processing. In Proceedings of the Middleware '19: 20th International Middleware Conference (pp. 215-227). ACM. Davis CA, USA.

Traub, J., Breß, S., Rabl, T., Katsifodimos, A., \& Markl, V. (2017, September). Optimized ondemand data streaming from sensor nodes. In Proceedings of the 2017 Symposium on Cloud Computing (pp. 586-597). ACM. Santa Clara, California, USA.

Watson, R.M., Gross, J.N., Taylor, C.N., \& Leishman, R.C. (2020). Robust incremental state estimation through covariance adaptation. IEEE Robotics and Automation Letters, 5(2), 3737 3744.

Xu, C., Yang, H.H., Wang, X., \& Quek, T.Q.S. (2019). Optimizing information freshness in computing-enabled IoT networks. IEEE Internet of Things Journal, 7(2), 971-985.

Yan, G., Tien, D., \& Sun, X. (2018, December). A kind of distributed fusion incremental kalman filter. In Proceedings of 2018 26th International Conference on Systems Engineering, (pp. 13). IEEE. Sydney, Australia.

Zhao, B., Hung, N.Q.V., \& Weidlich, M. (2020, April). Load shedding for complex event processing: input-based and state-based techniques. In 2020 IEEE 36th International Conference on Data Engineering (ICDE) (pp. 1093-1104). IEEE. Dallas, TX, USA. 